\title{
REVISÃO TAXONÔMICA DO GÊNERO HOMODIAETUS (TELEOSTEI, SILURIFORMES, TRICHOMYCTERIDAE)
}

\author{
Walter Rudolf Koch ${ }^{1}$
}

\begin{abstract}
TAXONOMIC REVISION OF GENUS HOMODIAETUS (TELEOSTEI, SILURIFORMES, TRICHOMYCTERIDAE). The genus Homodiaetus Eigenmann \& Ward, 1907 is revised and four species are recognized. Its distribution is restricted to southeastern South America, from Uruguay to Paraguay river at west to the coastal drainages of Rio de Janeiro State, Brazil. Homodiaetus is currently distinguished from other genus of Stegophilinae by the combination of the following characters: origin of ventral-fin at midlength between the snout tip and the caudal-fin origin; opercle with three or more odontodes; and gill membranes confluent with the istmus. Homodiaetus anisitsi Eigenmann \& Ward, 1907 , is diagnosed by the caudal-fin with black middle rays, margin of upper and lower procurrent caudal-fin rays with dark stripes extending to the caudal-fin, and 3-6 opercular odontodes; $H$. passarellii (Ribeiro, 1944) with 6-7 opercular odontodes, 21-24 lower procurrent caudal-fin rays and 23-26 upper procurrent caudal-fin rays; $H$. banguela sp. nov. with 9 opercular odontodes, 17-19 lower procurrent caudal-fin rays, 17-22 upper procurrent caudal-fin rays, reduction of fourth pharyngobranchial with only three teeth and untoothed fifth ceratobranchial; and H. graciosa sp. nov. with 5-6 dentary rows, 7-9 opercular odontodes and 16-23 upper procurrent caudal-fin rays.
\end{abstract}

KEYWORDS. Homodiaetus, Stegophilinae, Trichomycteridae, Neotropical, Taxonomy.

\section{INTRODUÇÃO}

A ordem Siluriformes compreende cerca de 34 famílias, 412 gêneros e mais de 2405 espécies. Destas, aproximadamente 1440 habitam o novo mundo (NELSON, 1994).

Entre as famílias pertencentes a esta ordem, Trichomycteridae apresenta a maior área de distribuição na América do Sul, estendendo-se do Panamá até o Chile e Argentina. Atualmente, são reconhecidas oito subfamílias de tricomicterídeos, formadas em sua maioria por espécies de vida livre, apesar de serem conhecidos como bagres-parasitas devido aos hábitos de algumas espécies de Vandellinae e Stegophilinae, que incluem bagres comumente chamados de candirus, conhecidos popularmente na Amazônia e arredores por penetrar ocasionalmente na uretra humana (PINNA \& BRITSKI, 1991).

A subfamília Stegophilinae é amplamente distribuída pelas principais bacias hidrográficas sul-americanas, incluindo Amazonas, Orinoco, São Francisco, ParanáParaguai e aquelas do sul do Brasil. Apesar de sua diversidade e do grande interesse

1.Universidade do Vale do Rio dos Sinos, Laboratório de Ecologia de Peixes, Av. UNISINOS, 950, 93022-000, São Leopoldo, RS, Brasil. (wkoch@bios.unisinos.br) 
ecológico e comportamental, a sistemática dos Stegophilinae é pouco estudada, segundo EigenMann (1918), Myers (1944) e Ribeiro (1951). Este último, em análise da filogenia do grupo, considerou todos os gêneros definidos em bases não-filogenéticas.

Os Stegophilinae compreendem cerca de 10 gêneros, diagnosticados pela ausência de barbilhão nasal e mental. Possuem dois pares de barbilhões maxilares no ângulo da boca, sendo o par inferior, pequeno. A boca é larga e inferior, com numerosos dentes dispostos em várias faixas regulares por toda sua margem. Os dentários estão em contato na sínfise mandibular e o opérculo e interopérculo possuem odontódeos (BURGESS, 1989). O gênero Homodiaetus foi proposto por EIGENMANN \& WARD (1907), tendo como espécietipo Homodiaetus anisitsi, procedente de um pequeno arroio em Vila Rica, Paraguai. Steindachner (1879) descreveu Stegophilus maculatus da bacia do rio da Prata, que EigenMANN (1910) colocou no gênero Henonemus Eigenmann \& Ward, 1907 e, na sua revisão da família em 1918, transferiu-o para o gênero Homodiaetus. Devincenzi \& VAZFERREIRA (1939) citaram a ocorrência de duas espécies de Homodiaetus no rio Uruguai, $H$. maculatus e H. vazferreirai Devincenzi, 1939, que difere da espécie-tipo do gênero por possuir seis filas superiores de dentes e seis inferiores, além de sete espinhos operculares e 8-12 interoperculares. Myers (1942) descreveu H. haemomyzon, encontrada no rio Guarico, Venezuela, que difere de $H$. anisitsi pelo número reduzido de raios procorrentes caudais, pela posição posterior da nadadeira ventral e pela coloração.

MyERS (1944), ao estudar a família Pygidiidae (Trichomycteridae), comentou que, devido às pequenas diferenças existentes entre Homodiaetus e Stegophilus Reinhardt, 1858 , é provável que o primeiro possa ser sinonimizado. Ribeiro (1944) descreveu Stegophilus passarellii, que BizerRIL (1994), através do exame de topótipos, concluiu tratar-se de uma espécie do gênero Homodiaetus. RiBeiro (1946), baseado no número e disposição dos barbilhões, assim como na presença de "eminências" no mento, redescreveu H. maculatus em um novo gênero, Parastegophilus. Fowler (1954), com base em levantamentos bibliográficos, registrou como válidas para o Brasil $H$. anisitsi e $H$. vazferreirai. RINGUELET et al. (1967), provavelmente desconhecendo o trabalho de RIBEIRO (1946), consideraram além de $H$. anisitsi e $H$. vazferreirai, H. maculatus como pertencente ao gênero. Posteriormente, essas espécies foram registradas por MenNi et al. (1984).

É apresentada a revisão taxonômica do gênero Homodiaetus, objetivando elucidar e subsidiar revisões sistemáticas mais abrangentes.

\section{MATERIAL E MÉTODOS}

Os exemplares estudados pertencem ao acervo das seguintes instituições (siglas, em sua maioria, conforme LeVITon et al., 1985): Academy of Natural Sciences, Philadelphia (ANSP), California Academy of Sciences, San Francisco (CAS), Field Museum of Natural History, Chicago (FMNH), Museu de Ciências e Tecnologia, Pontifícia Universidade Católica do Rio Grande do Sul, Porto Alegre (MCP), Museu de Ciências Naturais, Fundação Zoobotânica do Rio Grande do Sul, Porto Alegre (MCN), Museu de Zoologia da Universidade de São Paulo, São Paulo (MZSP), Museu Nacional, Universidade Federal do Rio de Janeiro, Rio de Janeiro (MNRJ), Museu Nacional de História Natural del Paraguay, Assunción (MNNP), Royal Ontario Museum, Toronto (ROM), University of Michigan Museum of Zoology, Ann Arbor (UMMZ) e National Museum of Natural History, Smithsonian Institution, Washington (USNM).

Foram realizadas cinco expedições de coleta, que abrangeram o sistema costeiro do sudeste (RJ), sistema costeiro do Estado do Paraná (PR), sistema do rio Uruguai (RS e SC) e sistema lagunar costeiro (RS). A nomenclatura empregada nas observações morfo-anatômicas do esqueleto segue BASKIN (1972), Arratia $(1982,1992)$, Castro \& Castro (1987), Pinna \& Britski (1991) e Reis (1998). Para o estudo osteológico, os exemplares foram preparados segundo a técnica de TAYlor \& VAN Dike (1985). Os 
espécimes foram digeridos por meio da enzima tripsina, ossos corados com vermelho de alizarina e cartilagens com azul de Alcian; após, passaram por uma série crescente de concentração de glicerina para diafanização, sendo posteriormente conservados em glicerina pura com timol. A dissecção dos espécimes seguiu, na maioria das vezes, a seguinte ordem: toda face esquerda, incluindo a série opercular, suspensório e mandíbula; a seguir, o aparelho branquial, incluindo o arco hióide, e as cinturas escapular e pélvica. A anatomia esqueletal foi examinada na seguinte ordem: pré-maxilar mediano, mesetmóide, vômer e pré-maxilar, palatino e maxilar, elementos dos arcos branquiais, suspensório e região opercular, neurocrânio, raios branquiostegais, nadadeira peitoral, nadadeira ventral, vértebras e esqueleto caudal.

Material comparativo examinado (conservado em álcool, Al; diafanizado, Di): Acanthopoma bondi Myers, 1942. EQUADOR, Napo: Coca (rio Napo, praia no meio do rio a montante da ponte), 3 Di (FMNH 96623). Acanthopoma sp. BRASIL, Amazonas: rio Negro (cachoeira do Bicho-Açu), 3 Al (MZSP 30417); Rondônia: (rio Madeira, na boca do rio Machado), 4 Al, 1 Di (MZSP 30421); Mato Grosso: Gaúcho do Norte (rio Xingú, encontro dos rios Culuene e Sete de Setembro), 1 Al (MZSP 46772). Henonemus punctatus (Boulenger, 1887). BRASIL, Pará: Caldeirão (rio Itacaiunas), $2 \mathrm{Al}$ (MZSP 30412). Henonemus sp. BRASIL, Goiás: Niquelândia (rio Maranhão no estreito, próximo à foz do rio Bagagem), 2 Al (MZSP 12749). Parastegophilus maculatus (Steindachner, 1879). BRASIL, São Paulo: Pirassununga, Cachoeira das Emas (rio Mogi-guaçú), 22 Al, 3 Di (MNRJ 4283). Parastegophilus sp. Piracicaba, 4 Al (MZSP 3110). Pseudostegophilus haemomyzon (Myers, 1942). VENEZUELA, Guarico: (rio Orituco), 18 Al, 3 Di (ANSP 137592); (rio Guarico), 1 Al, holótipo (CAS 136500); (rio Guarico), 1 Al, parátipo (CAS 136502). Pseudostegophilus maculatus (Steindachner, 1879). BRASIL, Rio Grande do Sul: Uruguaiana, 2 Al, 1 Di (FMNH 58526); Mato Grosso: (lagoas marginais à rodovia Transpantaneira), 1 Di (MZSP 38782). PARAGUAI, San Pedro: (rio AguarayGuazu), 4 Al, 2 Di (UMMZ 206662). BOLÍVIA, Beni: (rio Curiraba), 1 Al, 1 Di (USNM 305623). Pseudostegophilus nemurus (Günther, 1869). PERU, Loreto: (rio Amazonas ao sul da ilha Iquitos), 8 Al (ANSP 116448). EQUADOR, Napo: (praia arenosa do rio Payamino), 1 Di (FMNH 96625). PERU, Pebas: Shansho Caño (rio Ampiyacu), 1 Al (MNRJ 4220). BRASIL, Rondônia: (rio Madeira, boca do rio Machado), 4 Al, 2 Di (MZSP 30427). Pseudostegophilus scarificatus (Ihering, 1930). BRASIL, São Paulo: Porto Epitácio, 2 Al (MNRJ 4122); Pirassununga, Cachoeira das Emas (rio Mogi-Guaçú), 4 Al (MZSP 40237). Pseudostegophilus sp. BRASIL, Goiás: Minaçu-Cavalcante (rio Tocantins em Serra da Mesa), 1 Al (MNRJ 12595); São Paulo: Corumbataí (rio Corumbataí), 3 Al (MZSP 24834); Amazonas: Ati-Paraná, Fonte Boa Brasil (lago Buiu-Açu), 2 Al (MZSP 23427). Stegophilus insidiosus Reinhardt, 1858. BRASIL, Minas Gerais: (rio das Velhas), 1 Al (MCP 16609); Malhada (rio Abaeté, fazenda Macaúba), 2 Al (MZSP 39607); (Januaria), 1 Al (FMNH 58532).

A análise e o estudo da biometria e outros aspectos quantitativos das espécies consistiram: os 19 caracteres biométricos tomados em linha reta diretamente sobre o exemplar, sempre quando possível do seu lado esquerdo. Medidas do corpo foram feitas com paquímetro digital; para as da cabeça utilizou-se paquímetro sob estereomicroscópio. As seguintes medidas foram utilizadas para caracterizar as espécies: comprimento total, do ponto mais anterior da cabeça à extremidade da nadadeira caudal; comprimento padrão, do ponto mais anterior da cabeça à extremidade posterior do complexo uróforo; distância prédorsal, da ponta do focinho à base do primeiro raio da nadadeira dorsal; distância pré-ventral, da ponta do focinho à base do primeiro raio da nadadeira ventral; distância pré-peitoral, do focinho à base do primeiro raio da nadadeira peitoral; distância pré-anal, do focinho à base do primeiro raio da nadadeira anal; base da nadadeira dorsal, da base do primeiro raio à base do último raio; base da nadadeira anal, da base do primeiro raio à base do último raio; largura do corpo, medida à frente da nadadeira dorsal; altura do corpo, à frente da nadadeira dorsal; comprimento do pedúnculo caudal, da base do último raio da nadadeira anal à linha vertical que passa pela extremidade posterior do complexo uróforo; altura do pedúnculo caudal, no ponto mais estreito da altura do pedúnculo caudal; comprimento da cabeça, do focinho à extremidade do maior espinho opercular; altura e largura da cabeça, medidas na altura do interopérculo; distância interorbital, menor medida entre as bordas dos olhos; comprimento do focinho, da ponta do focinho ao início da borda do olho; distância das narinas anteriores, na margem interna das narinas; diâmetro do olho, medida longitudinal da órbita. O comprimento padrão está expresso em milímetros. Todas as demais medidas estão expressas como porcentagens do comprimento padrão e do comprimento da cabeça.

Foram realizadas 16 contagens sob estereomicroscópio: raios não-ramificados da nadadeira 
dorsal, raios ramificados da nadadeira dorsal, raios não-ramificados da nadadeira ventral, raios ramificados da ventral, raios não-ramificados da nadadeira peitoral, raios ramificados da nadadeira peitoral, raios não-ramificados da nadadeira anal, raios ramificados da nadadeira anal, raios da nadadeira caudal, raios procorrentes caudais superiores, raios procorrentes caudais inferiores, fileiras de dentes pré-maxilares, fileiras de dentes labiais, fileiras de dentes do dentário, espinhos do opérculo e espinhos do interopérculo. Os dados merísticos do holótipo aparecem nas descrições entre parênteses. Nas observações de $H$. anisitsi e $H$. passarellii esses valores não foram mencionados devido ao estado de conservação dos tipos. Os dados foram analisados estatisticamente com o software DATAX: Biometric data management system, v.4.2 de R. Reis \& N. F. Fontoura, 1993, não publicado.

$\mathrm{Na}$ lista de exemplares examinados de cada espécie, o número de espécimes em cada lote e o menor e o maior comprimento padrão $(\mathrm{CP})$ seguem o número de catálogo entre parênteses. Nessas listas, a procedência do material foi mantida como consta na etiqueta original que acompanha os exemplares, visando eliminar erros decorrentes de tradução.

\section{Homodiaetus Eigenmann \& Ward, 1907}

Homodiaetus EIgEnmann \& Ward, 1907:117 (espécie-tipo: Homodiaetus anisitsi Eigenmann \& Ward, 1907, por designação original e monotipia).

Os peixes incluídos no gênero Homodiaetus são pequenos (até $42,0 \mathrm{~mm}$ de comprimento padrão), translúcidos quando vivos, com exceção da cabeça e da região abdominal opacas, e compreendem quatro espécies distribuídas da foz da bacia do Prata até os rios costeiros do Rio de Janeiro (fig. 1).

Discussão. O gênero Homodiaetus é freqüentemente confundido com Acanthopoma, Henonemus e Stegophilus, mas pode ser distinguido por apresentar membranas branquiais confluentes com o istmo; em Acanthopoma as membranas são unidas entre si mas livres do istmo; três ou mais espinhos no opérculo ao invés de apenas dois em Henonemus; origem da nadadeira ventral situada quase no meio do corpo, considerando seu comprimento padrão, ao contrário de Stegophilus, onde a distância da origem das ventrais até a base da caudal é cerca de 1,5 a 2 vezes a distância entre ela e a ponta do focinho.

Os tipos de Homodiaetus haemomyzon, do rio Guarico, Venezuela, de maiores proporções, apresentam reduzido número de raios procorrentes caudais, posicionamento posterior das nadadeiras ventrais e coloração formando máculas no corpo (7-10), caracteres não encontrados em Homodiaetus, o que sugere a remoção desta espécie do gênero. Fazse necessária a revisão de Pseudostegophilus, que provavelmente incluirá esta espécie neste gênero, visto que sua descrição original apresenta observações errôneas, como a constatada por EIGENMANN (1918) e RIBEIRO (1951) em relação à presença de dois pares de barbilhões maxilares em vez de apenas um.

Diagnose. Homodiaetus distingue-se dos demais gêneros pela origem da nadadeira ventral eqüidistante da ponta do focinho e da origem da nadadeira caudal; presença de pelo menos três odontódeos operculares e membranas branquiais confluentes com o istmo.

Ecologia. Exemplares de Homodiaetus são encontrados em lagoas e rios arenosos de pouca profundidade. Freqüentemente são visualizadas escamas na boca e estômago desses peixes, confirmando o seu hábito lepidófago além de mucófago. Observações, em aquário, de exemplares de $H$. anisitsi revelaram seu parasitismo na medida em que se visualizou o bote destes peixes, arrancando escamas de espécimes das famílias Characidae e Cichlidae, além de perseguições a exemplares de Corydoras paleatus (Luiz R. Malabarba, comunic. pess.). Alguns Stegophilinae demonstram os mesmos hábitos, contudo não foram encontrados registros da fixação de Homodiaetus em outros peixes. 
Chave para as espécies de Homodiaetus.

1. Raios medianos da nadadeira caudal pretos, uma faixa escura superior e outra inferior prolongando-se sobre os respectivos raios procorrentes à região distal dos raios medianos da nadadeira caudal; 3-6 odontódeos operculares (rio Paraguai, baixo rio Paraná, rio Uruguai e sistema lagunar costeiro do Rio Grande do Sul). H. anisitsi Eigenmann \& Ward, 1907

Linhas escuras irregularmente dispostas na nadadeira caudal, sem o padrão acima descrito; 7-9 odontódeos operculares

2. Cinco a seis fileiras de dentes no dentário; 7-9 (usualmente 8) odontódeos operculares; 16-23 procorrentes superiores (rios costeiros do Paraná e rio Ribeira do Iguape, São Paulo).

H. graciosa sp. nov.

Seis a sete fileiras de dentes no dentário; 6,7 ou 9 odontódeos operculares (nunca 8); $17-26$ procorrentes superiores

3. Seis a sete odontódeos operculares; 21-24 raios procorrentes inferiores, 23-26 procorrentes superiores (rio Macacu, Rio de Janeiro)... H. passarellii (Ribeiro, 1944) Nove odontódeos operculares; 17-19 raios procorrentes inferiores, 17-22 procorrentes superiores (rio São João, Rio de Janeiro). H. banguela sp. nov.

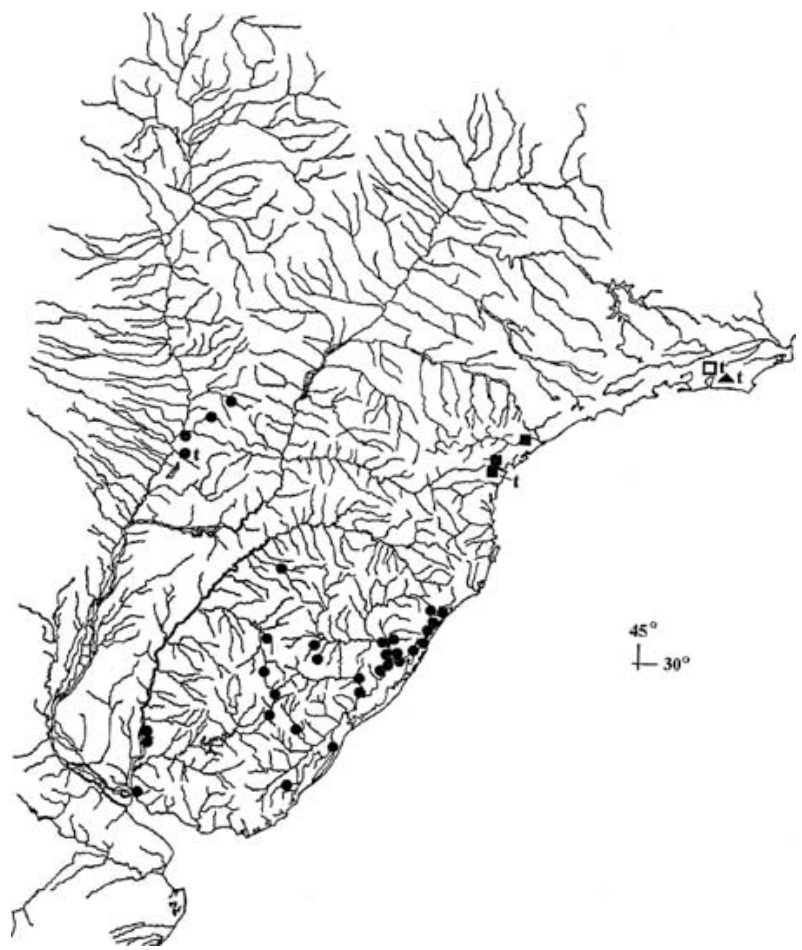

Fig. 1. Distribuição geográfica das espécies de Homodiaetus examinadas e respectivas localidades-tipo

(t) $(\bullet$, H. anisitsi Eigenmann \& Ward, 1907; $\square$, H. passarellii (Ribeiro, 1944); $\boldsymbol{\Delta}$, H. banguela sp. nov.; 口, H. graciosa sp. nov.). Alguns pontos representam mais de um lote. 


\section{Homodiaetus anisitsi Eigenmann \& Ward, 1907}

(Figs. 1, 2)

Homodiaetus anisitsi EIGENMAnN \& WARD, 1907:117 (localidade-tipo: Villa Rica, Paraguai).

Homodiaetus vazferreirai Devincenzi in Devincenzi \& Vaz-Ferreira, 1939:168 (localidade-tipo: Paysandú, Uruguai).

Material examinado. Holótipo de Homodiaetus anisitsi, PARAGUAI, Villa Rica (small creek at Villa Rica), J.D. Anisits (CAS 37276, 1 Al, 34,9mm CP). PARAGUAI, Caaguazu: (arroyo Guazu at bridge on hwy), 03.VIII.1979, J.N. Taylor et al. (UMMZ 206897, 3 Al, 23,8-34,2mm CP); Canendiyu: (arroyo Carimbatay ca. 15,6 Km WSW by dirt road of Curuguaty), 06.VII.1979, R.M. Bailey et al. (UMMZ 206292, $4 \mathrm{Al}, 1 \mathrm{Di}, 26,1-33,5 \mathrm{~mm} \mathrm{CP}$ ); (rio Jejuy, trib. to rio Jejui-Guazu ca. 40 Km N of Curuguaty), 07.VII.1979, R.M. Bailey \& J.N. Taylor (UMMZ 206341, 4 Al, 2 Di, 27,431,4mm CP). BRASIL, Rios costeiros do nordeste do Rio Grande do Sul: Osório (Lagoa da Pinguela, km 78 da BR-101), 01.IV.1990, B.S. Dyer \& A. Ramirez (MCP 13882, 3 Al, 23,4-28,5mm CP); Maquiné (junto à ponte da BR-101), 27.I.1983, C.A.S. Lucena \& Z.M. Lucena (MCP 15457, $1 \mathrm{Al}, 32,8 \mathrm{~mm}$ CP); (rio Maquiné junto à ponte da BR-101), 27.I.1983, C.A.S. Lucena \& Z.M. Lucena (MCP 9107, 1 Al, 33,2mm CP); Terra de Areia (rio Cornélios, estrada Praia do Barco/Terra de Areia), 15.XII.1992, P.H. Wimberger, R.E. Reis \& C.S. Fontana (MCP 16183, 9 Al, 25,1-35,0mm $\mathrm{CP}$ ); (foz do rio Maquiné, Lagoa dos Quadros, Estação Experimental de Piscicultura da Lagoa dos Quadros) (MCP 10465, 10 Al, 27,4-36,9mm CP); (Lagoa Itapeva), 26.VIII.1995, N.F. Fontoura (MCP 18899, 1 Al, 29,5mm CP); 10.VIII.1996, N.F. Fontoura (MCP 18900, 5 Al, 28,3-34,6mm CP); (rio Sanga Funda, crossing the BR-101), 12.II.1988, R.M.C. Castro (USNM 297968, 9 Al, 19,8-23,3mm CP). Sistema da Laguna dos Patos: Viamão (rio Guaíba, ilha do Junco), 12.VIII.1990, A. Ramires (MCP 14181, 2 Di); (rio Guaíba, praia de Itapuã), III.1991, A.S. Jardim \& J.F. Pezzi da Silva (MCP 15995, 1 Al, 28,8mm CP); IX.1990, A.S. Jardim \& J.F. Pezzi da Silva (MCP 16009, 2 Al, 32,2-33,0mm CP); VI.1990, A.S. Jardim \& H. Heinrichs (MCP 16018, 6 Al, 21,8-33,4mm CP); VIII.1990, A.S. Jardim \& J.F. Pezzi da Silva (MCP 16019, 7 Al, 1 Di, 25,4-30,0mm CP); IV.90, A.S. Jardim, H. Heinrichs \& J.F. Pezzi da Silva (MCP 16020, 7 Al, 24,5-32,2mm CP); XII.1990, A.S. Jardim \& J.F. Pezzi da Silva (MCP 16021, 3 Al, 20,9-33,2mm CP); X.1990, A.S. Jardim \& J.F. Pezzi da Silva (MCP 16035, 12 Al, 24,7-35,9mm CP); (praia de Itapuã, near mouth of arroio Itapuã), 25.IV.1990, A.S. Jardim (ANSP 168835, 7 Al, 25,1-28,4mm CP); São Lourenço do Sul (arroio da viúva Tereza), 05.V.1987, C.A.S. Lucena, L.R. Malabarba \& E.H. Pereira (MCP 11100, 5A1, 24,831,3mm CP); Barra do Ribeiro (Açude dos Garcia), C.A. Lucena, R.E. Reis \& T. Schultz (MCP 11118, $25 \mathrm{Al}, 3 \mathrm{Di}, 25,6-38,0 \mathrm{~mm} \mathrm{CP}$ ); Jaguarão (foz do rio Telho com o rio Jaguarão), 07.I.1987, P. Azevedo \& I.A. Costa (MCP 11175, 5 Al, 33,3-34,2mm CP); São Sepé (rio São Sepé, próximo à ponte da estrada São Sepé/São Rafael), J.F. Pezzi da Silva, A. R.Cardoso \& A. Ramires (MCP 17263, 7 Al, 23,3-26,8mm CP); Porto Alegre (rio Jacuí, Saco da Alemoa), 04.XII.1995, J.F. Pezzi da Silva \& F. Becker (MCP 18407, 5 Al, 30,7-35,8mm CP); Camaquã (rio Camaquã e poças laterais), 05.V.1987, C.A.S. Lucena, L.R. Malabarba \& E.H. Pereira (MCP 11420, 3 Al, 27,3-29,4mm CP); Caçapava do Sul (arroio Santa Bárbara, $12 \mathrm{Km}$ a oeste da Vila do Segredo), 10.X.1993, J.F. Pezzi da Silva et al. (MCP 16609, 3 Al, 2 Di, 23,3-35,5mm CP); Rio Grande (Lagoa Nicola), 17.I.1986, S.D. Hahn \& K.M. Grosser (MCN 7694, 4 Al, 22,5-24,0mm CP). URUGUAI, Rocha: (rio Cebollati, Paso de Averias), 21.XI.1926, C.C. Sanborn (FMNH 71371, 6 Al, 27,3-37,1mm CP). Rio Uruguai: URUGUAI, Soriano: (praia La Agraciada), 15.IV.1984, G. Skuk (MZSP 45701, 3 Al, 33,8-34,8mm CP); (mouth of Isla Queguay Grande), 1943, G.W. Teague (CAS 76893, 1 Al, 35,8mm CP); Cerro Largo: Arreria (lagoa a $10 \mathrm{Km}$ do rio Negro), 19.IV.1984, C.A.S. Lucena \& P. Chagas Pinto (MCP 9987, 6 Al, 1 Di, 28,6-41,7mm CP). BRASIL, Rio Grande do Sul: Santana do Livramento, (arroio Ibicuí da Faxina, próximo à BR-158), 02.IV.1993, J.F. Pezzi da Silva, E.H.L. Pereira \& A. Ramires (MCP 16157, 7 Al, 2 Di, 24,6-31,1mm CP); São Luis Gonzaga, (arroio Xinbocuzinho, estrada São Luis Gonzaga/Bossoroca), 11.XII.1992, P.H. Wimberger, R.E. Reis \& J.F. Pezzi da Silva (MCP 16185, 1 Al, 42mm CP); Bagé, (rio Negro a 14 Km de Bagé, em direção a Aceguá), 09.XII.1992, P.H. Wimberger, R.E. Reis \& J.F. Pezzi da Silva (MCP 16188, 6 Al, 2 Di, 29,7-38,5mm CP); Cacequi (rio Saicã), 13.IX.83, C.A.S. Lucena, L.R. Malabarba \& R.E. Reis, (MCP 9353, 6 Al, 30,1-39,6mm CP). 

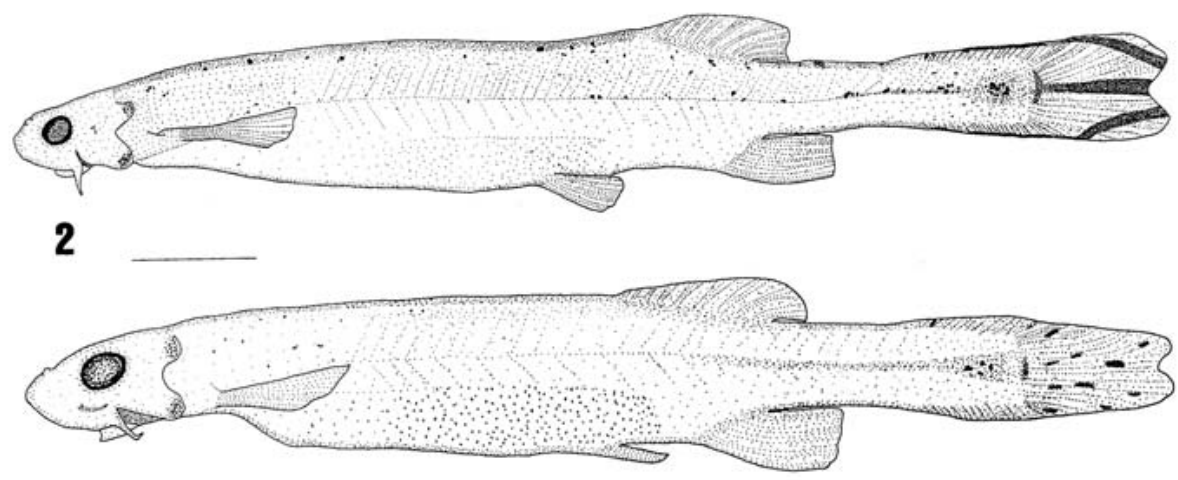

3

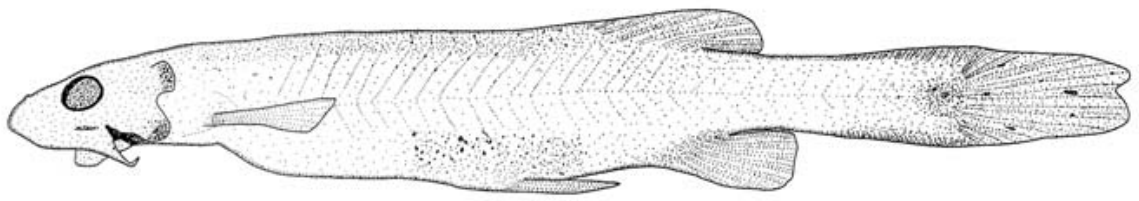

4

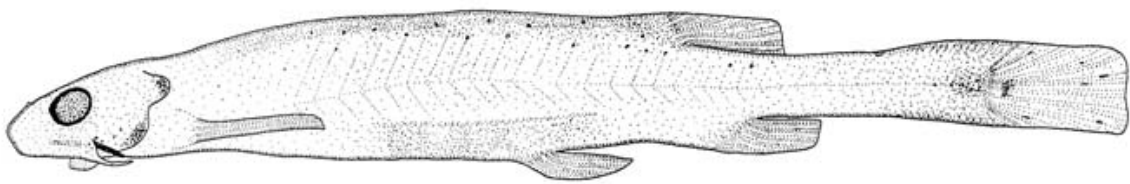

5

Figs. 2-5. Homodiaetus, vista lateral: 2, H. anisitsi Eigenmann \& Ward, 1907, MCP 16185; 3, H. passarellii (Ribeiro, 1944), MCN 12707; 4, H. banguela sp. nov., holótipo, MCP 19619; 5, $H$. graciosa sp. nov., holótipo, MCP 19618. Barras, $5 \mathrm{~mm}$, exceto fig. 2, barra, $10 \mathrm{~mm}$.

Diagnose. Homodiaetus anisitsi caracteriza-se pelos raios medianos da nadadeira caudal pretos e pelas faixas escuras, uma superior e outra inferior, prolongadas sobre os respectivos raios procorrentes à região distal dos raios medianos da nadadeira caudal (fig. 2). Outro caráter útil para distingui-la das demais espécies é a presença de 3-6 odontódeos operculares.

Descrição. Corpo delgado, cabeça deprimida, quase tão larga quanto longa, pedúnculo caudal comprimido. Perfil superior da cabeça levemente convexo, com tênue curvatura do focinho à origem da nadadeira dorsal; perfil da base da nadadeira dorsal quase reto até a origem da nadadeira caudal; perfil ventral da cabeça e corpo retos. Focinho de contorno arredondado a achatado, com dois pares de narinas tubulares, as anteriores mais proeminentes e mais distantes entre si que as posteriores. Olhos súperolaterais, sem margem livre, dispostos de forma tal que suas bordas anteriores encontramse mais próximas entre si que as posteriores. Dois pares de barbilhões maxilares, ambos mais curtos que o comprimento do olho, o interno diminuto. Boca inferior, com dentes 
finos em forma de "colher"; lábio superior móvel, não protrátil, possuindo 3-4 fileiras de dentes livres do pré-maxilar, visíveis com a boca fechada; pré-maxilar com 4 e dentário com 4-6 fileiras de dentes. Opérculo com 3-6 odontódeos; interopérculo com 7-10. Membranas branquiais confluentes com o istmo. Origem da nadadeira dorsal atrás da origem da nadadeira ventral, eqüidistante da margem posterior do olho e da ponta da nadadeira caudal. Origem da nadadeira ventral aproximadamente eqüidistante do extremo anterior do focinho e da origem da nadadeira caudal. Origem da nadadeira anal sob o primeiro terço da nadadeira dorsal. Raios da nadadeira peitoral i,4-5; raios da nadadeira dorsal iii-vii,5-7; raios da nadadeira ventral ii,4; raios da nadadeira anal iii-vi,4-5; raios principais da nadadeira caudal $14-17$; $16-25$ raios procorrentes superiores que podem alcançar o extremo da nadadeira dorsal reclinada; $12-25$ raios procorrentes inferiores. Linha lateral curta, com dois poros, não alcançando o ápice da nadadeira peitoral (tab. I).

Coloração. Em álcool, amarelado com cromatóforos esparsos, maiores na região dorsal que no restante do corpo e na maioria das vezes ausentes ventralmente. Alguns exemplares apresentam uma mancha oblíqua lateral sob o olho, que não ultrapassa $o$ tamanho do mesmo. Base dos raios medianos da nadadeira caudal com uma pequena mácula vertical escura; alguns agrupamentos de cromatóforos, formando pequenas manchas, dispostas no dorso logo após a nadadeira dorsal; outros alinhados sobre o septo lateral horizontal dos miótomos, formando uma tênue linha escura no pedúnculo caudal. Raios medianos da nadadeira caudal pretos; faixa escura alongada na extremidade distal dos raios procorrentes prolongada em duas barras escuras oblíquas: uma superior, levemente curva para baixo, estendendo-se até a região distal dos raios medianos da nadadeira caudal e outra inferior, levemente curva para cima, estendendo-se até a região distal dos raios medianos da caudal.

Distribuição. Sistema do rio Paraguai, baixo rio Paraná, rio Uruguai e rios costeiros do Rio Grande do Sul (fig. 1).

\section{Homodiaetus passarellii (Ribeiro, 1944)}

(Figs. 1, 3, 6)

Stegophilus passarellii Ribeiro, 1944:1 (localidade-tipo: córrego do Barro Branco (Roncador), Magé, Rio de Janeiro).

Homodiaetus passareli; BIZERRIL, 1994:625 (topótipos).

Material examinado. Parátipos de Stegophilus passarellii. BRASIL, Rio de Janeiro: (córrego do Barro Branco ou Roncador), VI.1941, A. Passarelli Filho col. (MNRJ 3784, 3 Al, 29,8-29,9mm CP). Outros exemplares: Rio de Janeiro: Cachoeira de Macacu (rio Macacu), 15.XII.1992, C.R.S.F. Bizerril (MCP 18737, 2 Al, 1 Di, 27,7-31,3mm CP); (a jusante da Cachoeira de Macacu, RJ-122 próximo ao cemitério), 09.IV.1996, D.F. Moraes et al. col. (MCN 12707, 1 Al, 1 Di, 27,1-37,0mm CP); (MNRJ 14859, 2 Al, 33,9-37,3mm CP).

Diagnose. Homodiaetus passarellii distingue-se das demais espécies do gênero por: 6-7 fileiras de dentes no dentário; 6- 7 odontódeos operculares; 21-24 raios procorrentes inferiores e 23-26 superiores.

Descrição. Corpo delgado, cabeça deprimida, quase tão larga quanto longa, pedúnculo caudal comprimido (fig. 6). Perfil da cabeça levemente convexo, com tênue curvatura do focinho à origem da nadadeira dorsal; perfil da base da nadadeira dorsal quase reto até a origem da nadadeira caudal; perfil ventral da cabeça e corpo retos. 
Focinho dorsalmente arredondado a achatado, com dois pares de narinas tubulares, as anteriores mais proeminentes e com maior distância internasal que as posteriores. Olhos superiores sem margem livre e posicionados de forma que suas bordas anteriores encontram-se mais próximas entre si que as posteriores. Dois pares de barbilhões maxilares, ambos mais curtos que o comprimento do olho, o interno diminuto. Boca inferior; lábio superior móvel, não protrátil, com 4 fileiras de finos dentes em forma de "colher", livres do pré-maxilar, visíveis com a boca fechada; pré-maxilar com 4 e dentário com 6-7 fileiras de dentes. Opérculo com 6-7 odontódeos; interopérculo com 8-10. Membranas branquiais confluentes com o istmo. Origem da nadadeira dorsal atrás da origem da nadadeira ventral, eqüidistante da margem posterior do olho e da ponta da nadadeira caudal. Origem da nadadeira ventral aproximadamente eqüidistante do extremo anterior do focinho e da origem da nadadeira caudal. Origem da nadadeira anal sob o primeiro terço da nadadeira dorsal. Raios da nadadeira peitoral i,5; raios da nadadeira dorsal iv-v,6; raios da nadadeira ventral ii,4; raios da nadadeira anal iv-vi,4-5; raios principais da nadadeira caudal 14-15, 23-26 raios procorrentes superiores que alcançam o extremo da nadadeira dorsal reclinada, 21-24 raios procorrentes inferiores. Linha lateral curta, com dois poros, não alcançando o ápice da nadadeira peitoral (tab. II).

Coloração. Em álcool, amarelado com cromatóforos esparsos, maiores na região dorsal que no restante do corpo e na maioria das vezes não dispostos ventralmente. Mancha oblíqua lateral sob o olho, que não ultrapassa o tamanho do mesmo. Base dos raios da nadadeira caudal com pequena mácula vertical escura; agrupamentos de cromatóforos formando pequenas manchas no dorso, logo após a nadadeira dorsal; cromatóforos alinhados sobre o septo lateral horizontal dos miótomos. Linhas irregulares pretas dispostas sobre alguns raios da nadadeira caudal (fig. 3).

Tabela I. Dados morfométricos de Homodiaetus anisitsi.

\begin{tabular}{|c|c|c|c|c|c|}
\hline \multirow[b]{2}{*}{ Caráter } & \multirow[b]{2}{*}{$\mathrm{n}$} & \multicolumn{2}{|c|}{ amplitude } & \multirow[b]{2}{*}{ média } & \multirow{2}{*}{$\begin{array}{l}\text { Desvio } \\
\text { padrão }\end{array}$} \\
\hline & & menor & maior & & \\
\hline \multirow[t]{2}{*}{ Comprimento padrão(mm) } & 140 & 19,8 & 42,0 & 29,6 & 4,497 \\
\hline & \multicolumn{4}{|c|}{ Porcentagens do comprimento padrão } & \\
\hline Distância pré-dorsal & 140 & 57,9 & 71,9 & 61,6 & 1,844 \\
\hline Distância pré-ventral & 140 & 46,2 & 59,8 & 50,2 & 1,845 \\
\hline Distância pré-peitoral & 140 & 13,0 & 19,7 & 16,8 & 1,126 \\
\hline Distância pré-anal & 140 & 59,9 & 74,5 & 64,8 & 2,216 \\
\hline Base da nadadeira dorsal & 140 & 8,2 & 14,2 & 11,2 & 1,100 \\
\hline Base da nadadeira anal & 139 & 8,9 & 14,9 & 11,4 & 1,083 \\
\hline Largura do corpo & 140 & 3,0 & 6,6 & 5,0 & 0,698 \\
\hline Altura do corpo & 140 & 8,8 & 14,1 & 11,4 & 1,101 \\
\hline Comprimento do pedúnculo caudal & 140 & 14,5 & 26,8 & 23,4 & 1,698 \\
\hline Altura do pedúnculo caudal & 140 & 5,3 & 7,8 & 6,5 & 0,574 \\
\hline \multirow[t]{2}{*}{ Comprimento da cabeça } & 140 & 14,1 & 19,2 & 16,1 & 0,883 \\
\hline & \multicolumn{4}{|c|}{ Porcentagens do comprimento da cabeça } & \\
\hline Altura da cabeça & 140 & 37,8 & 60,0 & 47,4 & 4,488 \\
\hline Largura de cabeça & 140 & 70,3 & 101,6 & 86,5 & 5,331 \\
\hline Distância interorbital & 140 & 15,7 & 32,8 & 22,2 & 2,634 \\
\hline Comprimento do focinho & 139 & 14,3 & 34,5 & 21,8 & 3,177 \\
\hline Distância das narinas anteriores & 135 & 15,6 & 25,0 & 19,5 & 1,738 \\
\hline Diâmetro do olho & 140 & 24,1 & 35,3 & 30,0 & 2,075 \\
\hline
\end{tabular}




\title{
Distribuição. Rio Macacu, Estado do Rio de Janeiro (fig. 1). \\ Homodiaetus banguela sp. nov.
}

\author{
(Figs. 1, 4, 7)
}

Material-tipo. Holótipo, BRASIL, Rio de Janeiro: Silva Jardim, (rio São João, no cruzamento com a BR-101), 18.XII.1992, C.R.S.F. Bizerril col. (MCP 19619, 31,8mm CP). Parátipos, coletados com o holótipo (MCP 18736, 3 Al, 1 Di, 14,9-29,9mm CP).

Diagnose. Homodiaetus banguela caracteriza-se pela redução do quarto faringobranquial, com número pequeno de dentes (três no exemplar diafanizado) e quinto ceratobranquial sem dentes (fig. 7). A combinação de outros caracteres são úteis para distinguir $H$. banguela das demais espécies do gênero: 6 fileiras de dentes no dentário; 9 odontódeos operculares; 17-19 raios procorrentes inferiores e 17-22 raios superiores.

Descrição. Corpo delgado, cabeça deprimida, quase tão larga quanto longa, pedúnculo caudal comprimido. Perfil da cabeça levemente convexo, com pequena curvatura do focinho à origem da nadadeira dorsal; perfil da base da nadadeira dorsal quase reto até a origem da nadadeira caudal; perfil ventral da cabeça e corpo retos. Focinho de contorno arredondado a achatado, com dois pares de narinas tubulares, as anteriores mais proeminentes e com maior distância internasal que as posteriores. Olhos superiores sem margem livre e posicionados de forma que suas bordas anteriores encontram-se mais próximas entre si que as bordas posteriores. Dois pares de barbilhões maxilares, ambos mais curtos que o comprimento do olho, o interno diminuto. Boca inferior; lábio superior móvel, não protrátil, com 4 fileiras de finos dentes em forma de "colher", livres do prémaxilar, visíveis com a boca fechada; pré-maxilar com 4 e dentário com 6 fileiras de dentes. Opérculo e interopérculo com 9 odontódeos cada. Membranas branquiais confluentes com o istmo. Origem da nadadeira dorsal atrás da origem da nadadeira ventral, eqüidistante

Tabela II. Dados morfométricos de Homodiaetus passarellii.

\begin{tabular}{|c|c|c|c|c|c|}
\hline \multirow[b]{2}{*}{ Caráter } & \multirow[b]{2}{*}{$\mathrm{n}$} & \multicolumn{2}{|c|}{ amplitude } & \multirow[b]{2}{*}{ média } & \multirow{2}{*}{$\begin{array}{l}\text { Desvio } \\
\text { padrão }\end{array}$} \\
\hline & & menor & maior & & \\
\hline \multirow[t]{2}{*}{ Comprimento padrão (mm) } & 7 & 27,1 & 37,3 & 32,2 & 4,109 \\
\hline & \multicolumn{5}{|c|}{ Porcentagens do comprimento padrão } \\
\hline Distância pré-dorsal & 7 & 60,0 & 63,9 & 62,1 & 1,418 \\
\hline Distância pré-ventral & 7 & 50,8 & 52,1 & 51,5 & 0,527 \\
\hline Distância pré-peitoral & 7 & 16,4 & 18,5 & 17,4 & 0,665 \\
\hline Distância pré-anal & 7 & 62,9 & 67,4 & 65,5 & 1,579 \\
\hline Base da nadadeira dorsal & 7 & 10,8 & 13,1 & 11,8 & 0,901 \\
\hline Base da nadadeira anal & 7 & 9,4 & 12,1 & 10,8 & 1,056 \\
\hline Largura do corpo & 7 & 4,5 & 5,9 & 5,2 & 0,473 \\
\hline Altura do corpo & 7 & 12,2 & 15,0 & 13,4 & 1,023 \\
\hline Comprimento do pedúnculo caudal & 7 & 21,8 & 24,9 & 23,4 & 1,273 \\
\hline Altura do pedúnculo caudal & 7 & 6,2 & 8,6 & 7,2 & 0,795 \\
\hline \multirow[t]{2}{*}{ Comprimento da cabeça } & 7 & 17,0 & 18,8 & 17,8 & 0,745 \\
\hline & \multicolumn{5}{|c|}{ Porcentagens do comprimento da cabeça } \\
\hline Altura da cabeça & 7 & 43,5 & 47,5 & 45,4 & 1,340 \\
\hline Largura da cabeça & 7 & 86,3 & 91,3 & 88,8 & 1,719 \\
\hline Distância interorbital & 7 & 21,6 & 28,1 & 25,1 & 2,226 \\
\hline Comprimento do focinho & 7 & 23,2 & 27,0 & 24,4 & 1,516 \\
\hline Distância das narinas anteriores & 7 & 17,4 & 21,4 & 19,7 & 1,669 \\
\hline Diâmetro do olho & 7 & 25,0 & 30,4 & 26,6 & 1,924 \\
\hline
\end{tabular}


Tabela III. Dados morfométricos de Homodiaetus banguela.

\begin{tabular}{|c|c|c|c|c|c|}
\hline \multirow[b]{2}{*}{ Caráter } & \multirow[b]{2}{*}{$\mathrm{n}$} & \multicolumn{2}{|c|}{ amplitude } & \multirow[b]{2}{*}{ média } & \multirow{2}{*}{$\begin{array}{l}\text { Desvio } \\
\text { padrão }\end{array}$} \\
\hline & & menor & maior & & \\
\hline \multirow[t]{2}{*}{ Comprimento padrão(mm) } & 4 & 14,9 & 31,8 & 22,9 & 9,213 \\
\hline & \multicolumn{5}{|c|}{ Porcentagens do comprimento padrão } \\
\hline Distância pré-dorsal & 4 & 58,7 & 62,5 & 60,6 & 1,587 \\
\hline Distância pré-ventral & 4 & 49,0 & 53,1 & 50,7 & 1,912 \\
\hline Distância pré-peitoral & 4 & 18,0 & 20,8 & 18,9 & 1,309 \\
\hline Distância pré-anal & 4 & 59,3 & 65,7 & 62,8 & 2,871 \\
\hline Base da nadadeira dorsal & 4 & 11,3 & 12,9 & 11,9 & 0,734 \\
\hline Base da nadadeira anal & 4 & 10,4 & 12,8 & 11,4 & 1,115 \\
\hline Largura do corpo & 4 & 2,7 & 6,0 & 4,5 & 1,466 \\
\hline Altura do corpo & 4 & 9,3 & 14,2 & 11,1 & 2,266 \\
\hline Comprimento do pedúnculo caudal & 4 & 21,7 & 26,7 & 23,8 & 2,135 \\
\hline Altura do pedúnculo caudal & 4 & 6,0 & 7,7 & 6,7 & 0,854 \\
\hline \multirow[t]{2}{*}{ Comprimento da cabeça } & 4 & 18,4 & 20,0 & 19,3 & 0,676 \\
\hline & \multicolumn{5}{|c|}{ Porcentagens do comprimento da cabeça } \\
\hline Altura da cabeça & 4 & 38,2 & 44,8 & 41,6 & 3,080 \\
\hline Largura da cabeça & 4 & 70,0 & 85,5 & 78,8 & 7,213 \\
\hline Distância interorbital & 4 & 20,0 & 22,6 & 20,8 & 1,220 \\
\hline Comprimento do focinho & 4 & 20,0 & 25,5 & 22,6 & 2,652 \\
\hline Distância das narinas anteriores & 4 & 13,3 & 21,0 & 17,4 & 3,157 \\
\hline Diâmetro do olho & 4 & 26,7 & 34,5 & 30,2 & 3,279 \\
\hline
\end{tabular}

Tabela IV. Dados morfométricos de Homodiaetus graciosa.

\begin{tabular}{|c|c|c|c|c|c|}
\hline \multirow[b]{2}{*}{ Caráter } & \multirow[b]{2}{*}{$\mathrm{n}$} & \multicolumn{2}{|c|}{ amplitude } & \multirow[b]{2}{*}{ média } & \multirow{2}{*}{$\begin{array}{r}\text { Desvio } \\
\text { padrão }\end{array}$} \\
\hline & & menor & maior & & \\
\hline \multirow[t]{2}{*}{ Comprimento padrão $(\mathrm{mm})$} & 23 & 21,0 & 35,4 & 26,6 & 4,236 \\
\hline & \multicolumn{4}{|c|}{ Porcentagens do comprimento padrão } & \\
\hline Distância pré-dorsal & 23 & 60,8 & 66,7 & 63,0 & 1,618 \\
\hline Distância pré-ventral & 23 & 47,1 & 53,4 & 50,7 & 1,660 \\
\hline Distância pré-peitoral & 23 & 16,5 & 19,3 & 17,6 & 0,844 \\
\hline Distância pré-anal & 23 & 62,6 & 67,8 & 65,5 & 1,530 \\
\hline Base da nadadeira dorsal & 23 & 9,7 & 13,9 & 12,0 & 1,059 \\
\hline Base da nadadeira anal & 23 & 9,6 & 14,8 & 11,5 & 1,201 \\
\hline Largura do corpo & 23 & 4,0 & 6,2 & 4,9 & 0,554 \\
\hline Altura do corpo & 23 & 10,7 & 14,3 & 12,1 & 1,039 \\
\hline Comprimento do pedúnculo caudal & 23 & 20,9 & 24,8 & 23,2 & 0,952 \\
\hline Altura do pedúnculo caudal & 23 & 6,3 & 7,8 & 6,9 & 0,429 \\
\hline \multirow[t]{2}{*}{ Altura da cabeça } & 23 & 16,8 & 19,2 & 17,9 & 0,584 \\
\hline & \multicolumn{4}{|c|}{ Porcentagens do comprimento da cabeça } & \\
\hline Altura da cabeça & 23 & 39,5 & 48,8 & 43,7 & 3,112 \\
\hline Largura da cabeça & 23 & 76,7 & 96,5 & 85,6 & 4,867 \\
\hline Distância interorbital & 23 & 18,2 & 26,3 & 21,6 & 2,134 \\
\hline Comprimento do focinho & 23 & 19,0 & 26,0 & 21,8 & 1,937 \\
\hline Distância das narinas anteriores & 22 & 17,5 & 22,8 & 19,5 & 1,398 \\
\hline Diâmetro do olho & 23 & 26,1 & 36,2 & 29,6 & 2,102 \\
\hline
\end{tabular}

da margem posterior do olho e da ponta da nadadeira caudal. Origem da nadadeira ventral aproximadamente eqüidistante do extremo anterior do focinho e da origem da nadadeira caudal. Origem da nadadeira anal sob o primeiro terço da nadadeira dorsal. Raios da nadadeira peitoral i,5(i,5); raios da nadadeira dorsal v-vi,5-6(vi,5); raios da nadadeira ventral ii,4(ii,4); raios da nadadeira anal iv,4(iv,4); raios principais da nadadeira caudal 1517 (17), 17-22 raios procorrentes superiores que alcançam o extremo da nadadeira dorsal 

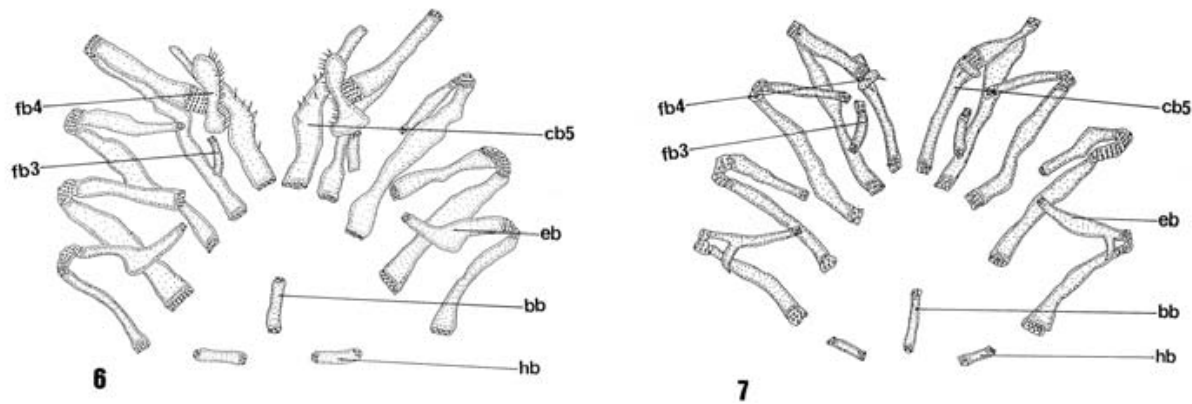

Figs. 6,7. Arcos branquiais, vista dorsal, região anterior para baixo: 6, Homodiaetus passarellii (Ribeiro, 1944), MCN 12707; 7, H. banguela sp. nov., parátipo, MCP 18736. As áreas com pontos representam ossos e as áreas com círculos cartilagens. Abreviaturas: fb, faringobranquial; cb, ceratobranquial; bb, basibranquial; eb, epibranquial; hb, hipobranquial. Barras, 0,25 mm.

reclinada, 17-19 raios procorrentes inferiores. Linha lateral curta, com dois poros, não alcançando o ápice da nadadeira peitoral (tab. III).

Coloração. Em álcool, amarelado com cromatóforos esparsos, maiores na região dorsal que no restante do corpo e na maioria das vezes não encontrados ventralmente. Mancha oblíqua lateral sob o olho, que não ultrapassa o tamanho do mesmo. Base dos raios da nadadeira caudal com pequena mácula vertical escura; agrupamentos de cromatóforos, formando pequenas manchas no dorso, após a nadadeira dorsal; cromatóforos alinhados sobre o septo lateral horizontal dos miótomos. Esparsas linhas irregulares pretas dispostas sobre os raios da nadadeira caudal (fig. 4).

Distribuição. Rio São João, Estado do Rio de Janeiro (fig. 1).

Etimologia. Banguela, substantivo em aposição, nome dado a desdentados, em alusão à ausência de dentes no quinto ceratobranquial.

\section{Homodiaetus graciosa sp. nov.}

(Figs. 1, 5)

Material-tipo. Holótipo, BRASIL, Paraná, Morretes, (rio Sagrado, posto florestal), 26.VII.1988, C.A.S. Lucena, L.R. Malabarba \& A. Bergmann, (MCP 19618, 33,5mm de CP). Parátipos: coletados com o holótipo (MCP 12844, 10 Al, 2 Di, 21,0-32,8mm de CP); Antonina (rio Mergulhão na estrada PR-340), 25.III.1995, J.M.R. Aranha, M.S. Menezes \& L.F. Favaro (MCP 19250, 2 Al, 29,7-35,4mm CP); Morretes (rio Nhundiaquara, expedição MZSP-USNM), 27-28.XII.1975, (MZSP 24806, 8 Al, 21,9-28,2mm CP); São Paulo, Juquiá, (ribeirão Poço Grande, rodovia SP-79, bacia do rio Ribeira do Iguape), 29.III.87, O.T. Oyakawa (MZSP 38613, 2 Al, 27,3-31,6mm CP).

Diagnose. Homodiaetus graciosa caracteriza-se por: 5-6 fileiras de dentes no dentário; 7-9 odontódeos operculares; 16-24 raios procorrentes inferiores e 16-23 superiores.

Descrição. Comprimento padrão dos espécimes examinados 21,0-35,4mm. Corpo delgado, cabeça deprimida, quase tão larga quanto longa, pedúnculo caudal comprimido. Perfil da cabeça levemente convexo, com tênue curvatura do focinho à origem da nadadeira dorsal; perfil da base da nadadeira dorsal quase reto até a origem da nadadeira caudal; 
perfil ventral da cabeça e corpo retos. Focinho arredondado a achatado, com dois pares de narinas tubulares, as anteriores mais proeminentes e com maior distância internasal que as posteriores. Olhos superiores sem margem livre e posicionados de forma que suas bordas anteriores encontram-se mais próximas entre si que as bordas posteriores. Dois pares de barbilhões maxilares, ambos mais curtos que o comprimento do olho, o interno diminuto. Boca inferior; lábio superior móvel, não protrátil, com 4 fileiras de finos dentes em forma de "colher", livres do pré-maxilar e visíveis com a boca fechada; pré-maxilar com 4 e dentário com 5-6 fileiras de dentes. Opérculo com 7-9 odontódeos; interopérculo com 8-12. Membranas branquiais confluentes com o istmo. Origem da nadadeira dorsal atrás da origem da nadadeira ventral, eqüidistante da margem posterior do olho e da ponta da nadadeira caudal. Origem da nadadeira ventral aproximadamente eqüidistante do extremo anterior do focinho e da origem da nadadeira caudal. Origem da nadadeira anal sob o primeiro terço da nadadeira dorsal. Raios da nadadeira peitoral i,5(i,5); raios da nadadeira dorsal iii-v,5-7(iv,5); raios da nadadeira ventral ii,4(ii,4); raios da nadadeira anal iv$\mathrm{v}, 4(\mathrm{iv}, 4)$; raios principais da nadadeira caudal 15-17 (15), 16-23 raios procorrentes superiores que alcançam o extremo da nadadeira dorsal reclinada, 17-24 raios procorrentes inferiores. Linha lateral curta, com dois poros, não alcançando o ápice da nadadeira peitoral (tab. IV).

Coloração. Em álcool, amarelado com cromatóforos esparsos, maiores na região dorsal que no restante do corpo e na maioria das vezes não encontrados ventralmente. Apresentam mancha oblíqua lateral sob o olho, que não ultrapassa o tamanho do mesmo. Base dos raios da nadadeira caudal com pequena mácula vertical escura; agrupamentos de cromatóforos formando pequenas manchas, encontrados no dorso, logo após a nadadeira dorsal; cromatóforos alinhados sobre o septo lateral horizontal dos miótomos. Linhas irregulares pretas dispostas sobre alguns raios da nadadeira caudal (fig. 5).

Distribuição. Rios costeiros do Estado do Paraná (Morretes, Paranaguá) e rio Ribeira do Iguape, Estado de São Paulo (fig. 1).

Etimologia. Graciosa, substantivo em aposição, alusivo a uma das áreas de ocorrência da espécie, Serra da Graciosa, Paraná.

Agradecimentos. A Roberto Esser dos Reis (MCP) pela orientação e leitura do manuscrito. Ao CNPq pela concessão da bolsa de Mestrado, junto ao curso de Pós-Graduação em Biociências, Zoologia, PUCRS. Aos pesquisadores Carlos A. S. Lucena, Luiz R. Malabarba, Zilda Margarete S. Lucena (MCP) e Mario C. C. de Pinna (MZSP), pela discussão de vários aspectos da biologia comparada e sugestões apresentadas. Aos ictiólogos e instituições de pesquisa, pelo empréstimo de exemplares e assistência: Scott A. Schaefer e William G. Saul (ANSP), Carl Ferraris (CAS), Mark Westneat e Mary Anne Rogers (FMNH), Karin M. Grosser (MCN), Dario Mandelburger (MNNP), Paulo A. Buckup e Décio F. de Moraes Jr. (MNRJ), Heraldo A. Britski, José L. Figueiredo, Naercio A. Menezes e Osvaldo Oyakawa (MZSP), Erling Holm (ROM), Richard Vari e Susan Jewett (USNM) e Douglas W. Nelson e William L. Fink (UMMZ). A Carlos Roberto S. F. Bizerril, Nelson F. Fontoura e Getúlio Dornelles de Souza pela doação de exemplares.

\section{REFERÊNCIAS BIBLIOGRÁFICAS}

Arratia, G. 1982. Esqueleto caudal de peces siluriformes y sus tendencias evolutivas (Diplomystidae y Trichomycteridae). Boln Mus. nac. Hist. nat., Santiago, 39:49-61.

1992. Development and variation of the suspensorium of primitive Catfishes (Teleostei: Ostariophysi) and their phylogenetic relationships. Bonn. zool. Monogr., Bonn, 32:1-149.

BizerRIL, C. R. S. F. 1994. Descrição de uma nova espécie de Trichomycterus (Siluroidei, Trichomycteridae) do Estado de Santa Catarina, com uma sinopse da composição da família Trichomycteridae no leste brasileiro. Arq. Biol. Tecnol., Curitiba, 37(3):617-628.

Burgess, W. E. 1989. An atlas of freshwater and marine catfishes a preliminary survey of the Siluriformes. Neptune, T.F.H. Publ. 784p. 
Castro, R. M. C. \& Castro, M. M. C. 1987. Proposta de uma nomenclatura osteológica para Characiformes (Pisces: Ostariophysi). Bolm Mus. para. Emilio Goeldi, Sér. Zool., Belém, 3(1):25-32.

Devincenzi, G. J. \& VAz-FerReira, R. 1939. Nota preliminar sobre un pygido hematófago del Rio Uruguay. Archos Soc. Biol. Montev., Montevideo, 9(3):165-78.

Eigenmann, C. H. 1910. Catalogue of the fresh water fishes of tropical and south temperate America. Rep. Princeton Univ. Exped. Patagonia., Princeton, 3(2):375-511.

. 1918. The pygidiidae, a family of South American catfishes. Mem. Carneg. Mus., Pittsburgh, 7(5):259-398.

Eigenmann, C. H. \& Ward, D. P. 1907. On further collections of fishes from Paraguay. Ann. Carneg. Mus., Pittsburgh, 4:110-17.

Fowler, H.W. 1954. Os peixes de água doce do Brasil. Archos Zool. Est. S. Paulo, São Paulo, 9(4):1-400.

Leviton, A. E.; Gibs, R. H. JR. et al. 1985. Standards in herpetology and ichthyology: part I. Standard symbolic codes for institutional resource collections in herpetology and ichthyology. Copeia, Austin, 1985(3):802-832.

Menni, R. C.; Lopez, H. L. et al. 1984. Ictiologia de áreas serranas de Cordoba y San Luis (Argentina). Biologia Acuatica, La Plata, (5):1-65.

Myers, G. S. 1942. Studies on South American fresh-water fishes. Stanford ichthyol. Bull., Stanford, 2(4):89-114.

1944. Two extraordinary new blind nematognath fishes from the rio Negro, representing a new subfamily of Pygidiidae, with a rearrangement of the genera of the family, and ilustrations of some previously described genera and species from Venezuela and Brazil. Proc. Calif. Acad. Sci., San Francisco, 23(40):591-602.

Nelson, J. S. 1994. Fishes of the world. New York, Wiley. 600p.

Pinna, M. C. C. DE \& BRiTski, H. A. 1991. Megalocentor, a new genus of parasitic catfish from the Amazon basin: the sister group of Apomatoceros (Trichomycteridae: Stegophilinae). Ichthyol. Explor. Freshwaters, München, 2 (2):113-28.

ReIs, R. E. 1998. Anatomy and phylogenetic analysis of the neotropical callichthyid catfishes (Ostariophysi, Siluriformes). J. Linn. Soc. Zoology, London, 124(2):105-168.

Ribeiro, P. M. 1944. Nova espécie para o gênero Stegophilus Reinhardt, 1858 ( Pisces Pygididae Stegophilinae). Bolm Mus. nac. Rio de J., Rio de Janeiro, (20):1-3.

1946. Notas para o estudo dos Pygidiidae brasileiros (Pisces - Pygidiidae - Stegophilinae). I. Bolm Mus. nac. Rio de J., Rio de Janeiro, (58):1-20.

1951. Notas para o estudo dos Pygidiidae brasileiros (Pisces - Pygidiidae - Stegophilinae). IV. Bolm Mus. nac. Rio de J., Rio de Janeiro, (106):1-16.

Ringuelet, R. A.; Aramburu, R. H. et al. 1967. Los peces argentinos de agua dulce. La Plata, Librart. $602 \mathrm{p}$.

SteindaChNer, F. 1879. Über einige neue und seltene Fish-Arten aus den k.k. Zool. Museum zu Wien, Stuttgart und Warschau (mit 9 Tafeln) - IV - Über einige neue und seltene Fish-Arten aus dem La Plata. Denkschr. Akad. Wiss., Wien, 41:1-52.

TAYLOR, W. R. \& VAN DiKe, G. C. 1985. Revised procedures for staining and clearning small fishes and other vertebrates for bone and cartilage study. Cybium., Paris, 9:107-119. 